\title{
Sarcoidosis in a young child with Alagille syndrome: a case report
}

\author{
Melissa Mannion ${ }^{1 *}$, Mem Zolak², David R Kelly ${ }^{3}$, Timothy Beukelman ${ }^{1}$ and Randy Q Cron ${ }^{1}$
}

\begin{abstract}
We report a now 6-year-old African-American male with both Alagille syndrome and pediatric sarcoidosis. With a prior JAG1 mutation positive diagnosis of Alagille syndrome, he presented to the hospital with a subacute, predominantly respiratory febrile condition, eventually diagnosed as sarcoidosis. A liver biopsy revealed paucity of bile ducts and scattered epithelioid granulomas, while a skin biopsy showed granulomatous angiitis, a manifestation of sarcoidosis not yet reported in a pediatric patient. He has subsequently been treated with corticosteroids, mycophenolate mofetil, and infliximab with clinical response. Alagille syndrome and sarcoidosis have not yet been reported in the medical literature in the same patient to the best of our knowledge. We briefly review these two seemingly unrelated conditions and propose a possible common pathogenic mechanism.
\end{abstract}

Keywords: Sarcoidosis, Alagille syndrome, Granulomatous angiitis, Granuloma, JAG1, NOTCH1, T lymphocyte

\section{Background}

Sarcoidosis is a systemic granulomatous disease of unknown etiology that may affect many organ systems. The lungs are the most commonly involved organs in adults. Adults with sarcoidosis often have lymphadenopathy and systemic manifestations, such as fever and weight loss. Diagnosis of pediatric sarcoidosis, as in adults, requires demonstration of non-caseating granulomata in one or more organs in the setting of consistent clinical or radiographic findings and the exclusion of infectious granulomatous conditions. There is no singular diagnostic lab test [1]. Derangement in calcium occurs in $30 \%$ of children with sarcoidosis and manifests as hypercalciuria, with or without hypercalcemia [2]. Hypercalcemia is more prevalent in affected children than adults. An elevated angiotensin converting enzyme (ACE) level can be found in sarcoidosis patients, however it is a less sensitive test in children than in adults and is thought to be a product of the epithelioid cells of the sarcoid granuloma [1]. In addition, serum lysozyme can be used as a marker for sarcoidosis. In one Japanese study, lysozyme had a sensitivity of $79 \%$ for predicting sarcoidosis. That study found that lysozyme levels

\footnotetext{
* Correspondence: mmannion@peds.uab.edu

${ }^{1}$ Department of Pediatrics, Division of Rheumatology, University of Alabama at Birmingham, Birmingham, AL, USA

Full list of author information is available at the end of the article
}

tended to increase with the number of organ systems involved and the disease activity [3].

Pediatric sarcoidosis is rare, and its true incidence is unknown. A recent Danish study estimates the incidence at $0.22-0.27$ per 100,000 children younger than 15 years of age but, different geographic regions or racial groups may have a higher prevalence [2]. Most cases of pediatric sarcoidosis are diagnosed in the pre-adolescent or adolescent age groups. By contrast, there is an earlyonset form that occurs in children up to age 4 or 5 years and is usually characterized by boggy arthritis, rash, and uveitis [4]. Onset frequently occurs in the first year of life, and the classic presentation is rash (usually papular and erythematous) or arthritis (usually a boggy tenosynovitis) without pulmonary involvement, followed by uveitis. Although pulmonary involvement is not typical at diagnosis, it can develop later in the disease course. Blau syndrome is a triad of synovitis, uveitis and rash with an autosomal dominant inheritance of a mutation in CARD15 (caspase recruitment domain)/NOD2 (nucleotide binding oligomerization domain) on chromosome $16[2,5]$ and may represent a substantial subset of what is called early-onset sarcoidosis. In addition to the familial Blau syndrome, there have been reports of sporadic mutations in CARD15 resulting in early onset sarcoidosis [6].
C Biomed Central

(c) 2012 Mannion et al.; licensee BioMed Central Ltd. This is an Open Access article distributed under the terms of the Creative Commons Attribution License (http://creativecommons.org/licenses/by/2.0), which permits unrestricted use, distribution, and reproduction in any medium, provided the original work is properly cited. 
In contrast, older children and adolescents with sarcoidosis are often more similar to adults with lung involvement and constitutional symptoms [1,2]. Almost half of children with sarcoidosis show restrictive lung disease on pulmonary function testing [2]. If there is pulmonary involvement, hilar adenopathy is a hallmark finding on chest radiograph $[1,2]$. Peripheral lymphadenopathy is also a common finding in older children with sarcoidosis, such that peripheral lymph node enlargement occurs in 40-70\% of children with sarcoidosis [2]. In addition, many children with sarcoidosis have cutaneous manifestations, such as erythema nodosum, papules, plaques, nodules, and changes in pigmentation [1]. Erythema nodosum can be seen in $31 \%$ of children with sarcoidosis [2]. Many children with sarcoidosis also have ocular involvement. Most commonly, patients have uveitis, but choroidal or conjunctival granulomata can also be seen [2]. Other organ systems, including the liver, can also be involved [1]. While genetic mutations in CARD15/NOD2 can be demonstrated in early onset sarcoidosis and Blau syndrome, there has not been a definitive genetic mutation associated with adult sarcoidosis or sarcoidosis in older children, and mutations in CARD15/NOD2 are not found in these patients [2].

Alagille syndrome is a heritable form of chronic liver disease in childhood associated with a mutation in JAG1. Patients with this syndrome have hypoplastic intrahepatic bile ducts with patent extrahepatic bile ducts [7-9] Other findings of the syndrome can include congenital heart disease, characteristic facial features, butterfly vertebrae, growth retardation, renal abnormalities, abnormal eye findings, pancreatic disease, and intracranial hemorrhage [7-9]. The most common form of cardiovascular involvement in Alagille syndrome is peripheral pulmonary artery stenosis. Liver biopsy reveals intrahepatic cholestasis in association with a relative paucity of intrahepatic interlobular bile ducts [7-9]. A prior association of Alagille syndrome and sarcoidosis has not been previously reported.

\section{Case presentation}

A 2-year-old male with a known history of genetically confirmed Alagille syndrome presented to the hospital with tachypnea, respiratory distress, and a fever of $104^{\circ} \mathrm{F}$. $\mathrm{He}$ had multiple hospitalizations in the preceding 3 months for similar symptoms and had been diagnosed with pneumonia 3 times and cholangitis once. He had recently finished a course of intravenous antibiotics for presumed pneumonia 2 days prior to presentation.

The patient's past medical history was significant for Alagille syndrome which presented as direct hyperbilirubinemia at 2 months of age. Liver biopsy was performed at 3 months and revealed cholestasis, paucity of bile ducts and giant cell transformation (Figure 1), consistent

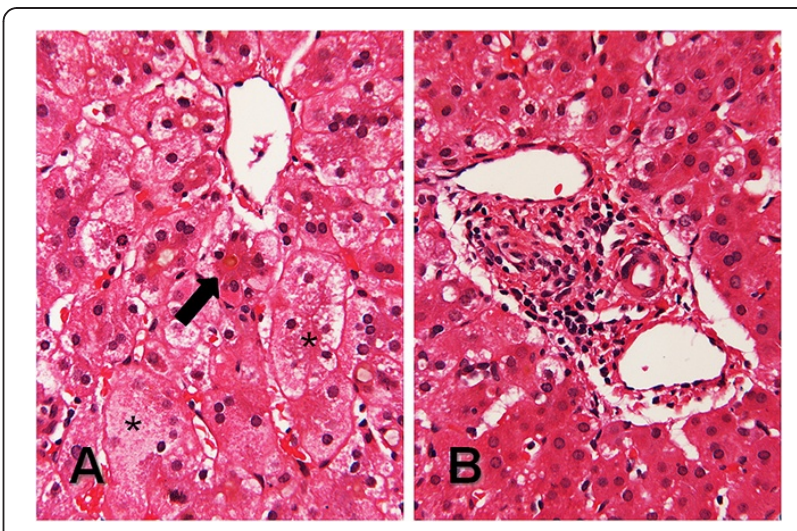

Figure 1 Liver biopsy at nine weeks consistent with Alagille syndrome. (A) Canalicular cholestasis (arrow) with scattered hepatic giant cells containing eight to ten nuclei (asterisks), and (B) a representative portal tract containing two small dilated veins, a small artery, and no recognizable bile ducts. (A and $\mathbf{B}$, hematoxylin and eosin, 132x original magnification).

with Alagille syndrome. He was also noted by imaging and clinical examination to have butterfly vertebrae, right and left pulmonary artery stenosis, and prominent forehead and eyes. The diagnosis was later confirmed by gene sequencing revealing that the patient had a novel $G$ to A point mutation in exon 20 of JAG1, which changes a tryptophan to a premature stop codon, a genetic result that would be consistent with the clinical diagnosis of Alagille syndrome. In addition, the child had severe failure to thrive. The child had a repeat liver biopsy (Figure 2) two months prior to presentation that revealed, in addition to signs of chronic liver disease with paucity of bile ducts and bridging fibrosis, several

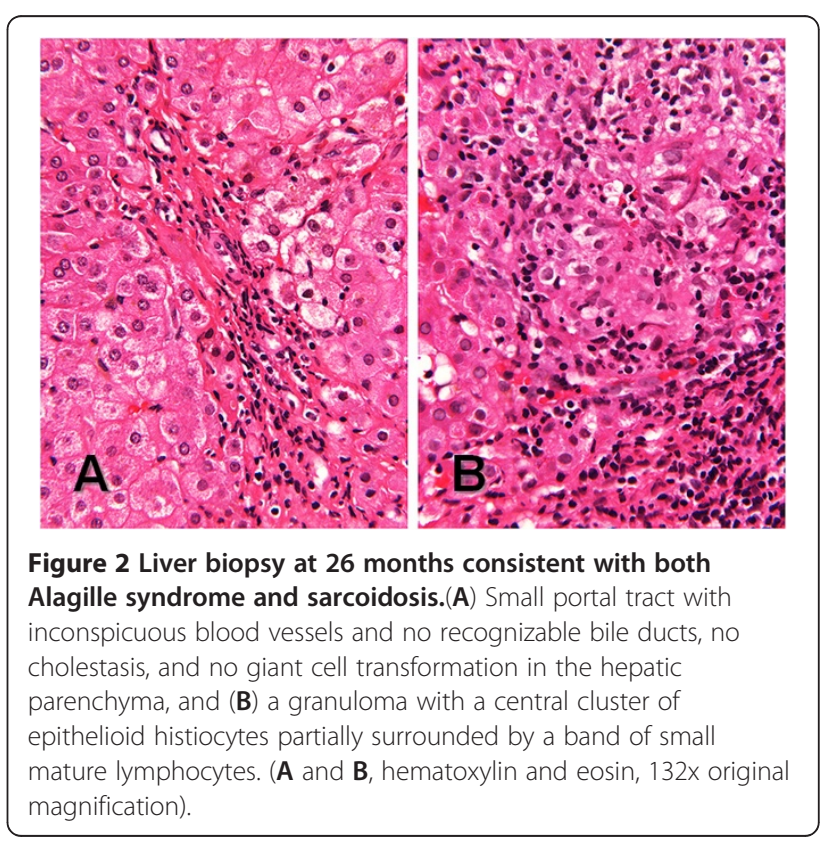


epithelioid cell granulomata (not typically a feature of Alagille syndrome).

On physical examination, the child had a temperature of $103^{\circ} \mathrm{F}$, a heart rate of 156 beats per minute, a blood pressure of $121 / 53 \mathrm{~mm} \mathrm{Hg}$, and had an oxygen requirement of $5 \mathrm{~L} /$ minute by face mask. He was tachypneic and grunting. He had shotty lymphadenopathy in his anterior and posterior cervical chains as well as in his inguinal region. His breath sounds were clear and equal bilaterally. He had a 3/6 systolic murmur heard best over the left upper sternal border. He had 2+ femoral and distal pulses, with $<2$ second capillary refill distally. The child's abdomen was soft and non-tender, with hepatomegaly, which was baseline for the patient. His spleen tip was not palpable. On the soles of the child's feet were multiple erythematous palpable nodules.

Initial laboratory studies revealed a white blood cell count of 19.6 thousand cells $/ \mu \mathrm{L}$, with $77 \%$ segmented neutrophils, $7 \%$ bands, $14 \%$ lymphocytes, and $2 \%$ monocytes. His hemoglobin level was $10 \mathrm{~g} / \mathrm{dL}$, and his platelets were $635,000 / \mu \mathrm{L}$. His electrolytes were all within normal limits, and his total serum protein was elevated at $8.9 \mathrm{~g} / \mathrm{dL}$, with an albumin of $4.2 \mathrm{~g} / \mathrm{dL}$.

The child was admitted to the pediatric intensive care unit for one day because of significant respiratory distress and started on intravenous broad spectrum antibiotics. The patient had an echocardiogram that was normal and revealed no vegetations. He had chest, abdominal, and pelvic computed tomography and radiography that failed to reveal an abscess or other obvious infectious process, but did show airspace and interstitial lung disease more on the right than left, including small areas with ground glass appearance, mediastinal and peritracheal adenopathy, butterfly vertebrae, and hepatomegaly (Figure 3). Abdominal ultrasound showed findings consistent with hepatic granulomata. All blood cultures were negative, and a bronchoalveolar lavage was performed that had no hemosiderin-laden macrophages and was negative for bacteria, fungi, pneumocystis, and mycobacteria. The patient had a negative tuberculosis skin test and 3 morning gastric aspirates that did not reveal mycobacteria.

Rheumatology was consulted when the infectious evaluation had failed to produce a diagnosis and his C-reactive protein and erythrocyte sedimentation rate (ESR) were elevated at $1.69 \mathrm{mg} / \mathrm{dL}$ and $80 \mathrm{~mm} / \mathrm{hr}$, respectively. An ophthalmologic examination revealed diffuse creamy white infiltrates and vascular sheathing consistent with vasculitis in both eyes. A skin biopsy of a nodule on the plantar aspect of his foot showed a granulomatous vascular reaction, consistent with granulomatous angiitis (Figure 4). A serum ACE level was $76 \mathrm{U} / \mathrm{L}$ (reference range: 13-100). The child's serum lysozyme level was elevated at $11.9 \mu \mathrm{g} / \mathrm{mL}$ (reference range: $4-10.3$ ). There was

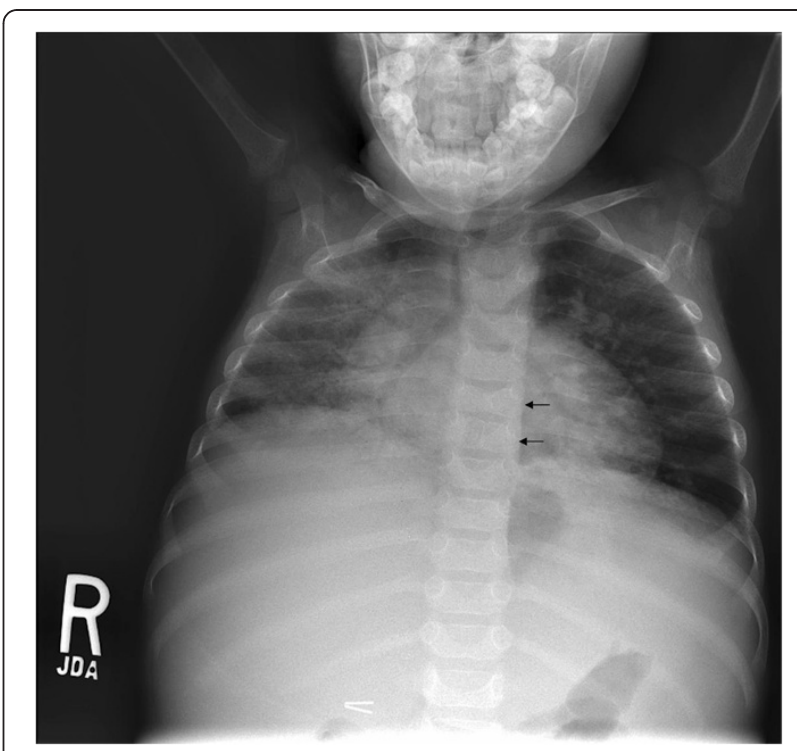

Figure 3 Chest radiograph consistent with Alagille syndrome and sarcoidosis. Anterior view chest radiograph revealing extensive bilateral chronic lung disease, mediastinal adenopathy, butterfly vertebrae (arrows), and hepatomegaly.

concern that the child may have been having some joint pains in his knees at home. Also, during his hospitalization, he indicated pain in his left elbow and had some mild joint swelling. Based on the constellation of findings and the granulomata on the liver biopsy performed 2 months prior, Rheumatology was concerned for sarcoidosis and recommended that the patient be treated with high dose glucocorticoids for 3 days $(30 \mathrm{mg} / \mathrm{kg} / \mathrm{day}$ of intravenous methylprednisolone). After the first dose, the child's tachypnea began to improve, his oxygen requirement resolved, and he became more active. Following the third dose, the child was started on $2 \mathrm{mg} / \mathrm{kg} /$ day of oral prednisolone.

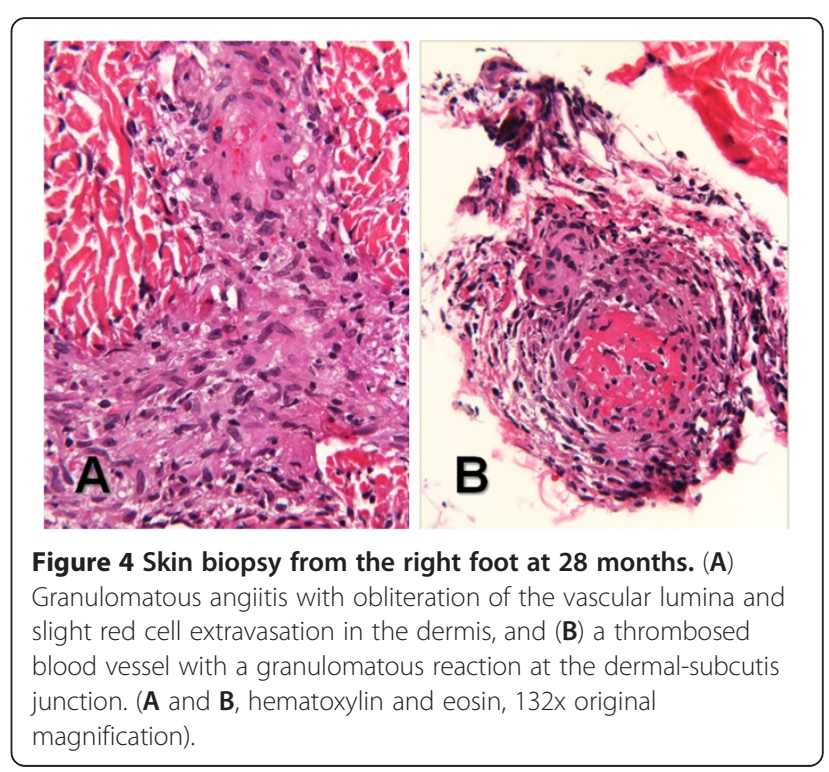


Mycophenolate mofetil $200 \mathrm{mg}$ twice a day was also initiated rather than methotrexate because of concern of additional liver toxicity in a child with Alagille syndrome, and the patient received an infusion of infliximab of $10 \mathrm{mg} / \mathrm{kg}$. The child's fevers, joint pains, tachypnea, oxygen requirement, and rash had resolved 4 days after starting pulse corticosteroids.

The child was discharged home on prednisolone ( $2 \mathrm{mg} / \mathrm{kg} /$ day) and mycophenolate mofetil with monthly infliximab infusions. One month following discharge, his ophthalmologic examination had returned to normal. The child initially did well, and a steroid dose wean and decrease in mycophenolate mofetil to $200 \mathrm{mg}$ once daily were initiated. Shortly after this change in medication dosages, the child began having fatigue, joint swelling, and an elevation in ESR. The child's infliximab dose was increased to $20 \mathrm{mg} / \mathrm{kg}$ and the dosing interval was shortened to 3 weeks. His prednisolone dose was increased until his next infliximab infusion. The child continued to not tolerate steroid weaning, so his infiximab infusion interval was decreased to 2 weeks and mycophenolate was returned to twice daily dosing to help decrease the steroid burden. The child has subsequently tolerated a steroid wean down to $0.2 \mathrm{mg} / \mathrm{kg} /$ day of prednisolone. His infliximab and methylprednisolone infusions have been spaced to every 3 weeks, and his arthritis, uveitis, and pleuritis have been under good control.

\section{Discussion}

The coexistence of Alagille syndrome and granulomatous disease has not been previously described in the literature. This case report highlights the potential for concurrent rare conditions in a single pediatric patient. Alternatively, these conditions may be related in an unrecognized way.

The diagnosis of inflammatory granulomatous disease in this patient was complicated by several factors. The patient's diagnosis of Alagille syndrome could explain some of his clinical features, such as hepatomegaly and failure to thrive [7]. In addition, there was a reluctance to consider two rare diagnoses in one patient [10]. While this 2-year-old child did have rash, arthritis, and eye findings, his pulmonary involvement, lymphadenopathy, and constitutional symptoms of fever and poor growth represent a presentation rarely seen in early-onset sarcoidosis [1]. The skin rash in this child clinically resembled erythema nodosum; however, morphologically, it consisted of granulomatous angiitis (with microthrombi) and primarily involved the dermis with no evidence of septal panniculitis. This cutaneous vascular manifestation of sarcoidosis is extremely rare in adults and not previously reported in children [11-14]. Vascular abnormalities, such as vessel wall abnormalities, aneurysms, vascular stenoses, moya moya, renovascular hypertension, and intracranial hemorrhage $[15,16]$ can be seen in Alagille syndrome, though diffuse granulomata consistent with sarcoidosis have not been described. A young girl with Takayasu arteritis and Alagille syndrome [15] represents the only known reported case of Alagille syndrome associated vasculitis.

Sarcoidosis patients may have hypercalcemia secondary to extrarenal production of calcitriol leading to increased intestinal absorption of calcium [17]. This child was being treated for hypocalcemia secondary to gastrointestinal losses due to his Alagille syndrome. Thus, it is possible that the child's Alagille syndrome masked a tendency toward hypercalcemia.

This child's atypical presentation of sarcoidosis and the rarity of his two conditions suggest a possible link between them. Although Alagille syndrome is not typically associated with granuloma formation, in one study, there was a patient with Alagille syndrome who developed a cystic mandibular mass, which on biopsy revealed multiple central giant cell granulomata that were nonmalignant [9]. This case causes speculation that perhaps granulomatous disease could be a manifestation of Alagille syndrome. Our patient had granulomata on a liver biopsy during a previous evaluation 2 months prior to presentation, which at the time of examination, had been attributed to his Alagille syndrome. Recent reviews of hepatic granulomas report an incidence of approximately $4 \%$ in unselected liver biopsies in adults. The causation of hepatic granuloma can be determined in most cases and varies by region; noninfectious immune categories predominate in Western countries, where infectious causes predominate in the developing world [18]. Nevertheless, for this child, the constellation of uveitis, arthritis, pleuritis, and rash in the setting of an elevated ACE level and granulomas on biopsy strongly argue in favor of coincident diagnosis of sarcoidosis.

There may be a molecular basis for our patient's constellation of findings. The two genes associated with Alagille syndrome are JAG1 and NOTCH2 [16]. JAG1 encodes a cell surface ligand for one of the Notch transmembrane receptors. The role of the Notch signaling proteins is not well understood, but is highly conserved; JAG1 is on chromosome 20 and accounts for $70 \%$ of the gene mutations in Alagille syndrome [7]. There are four mammalian Notch receptors (1-4) and five Notch ligands (Jagged1, Jagged2, Delta-like 1, Delta-like 3, and Delta-like 4) [19]. Of note, JAG1 mutations are not specific for Alagille syndrome [16]; in a large kindred study, JAG1 mutations were associated with congenital right heart obstructive disease without other diagnostic criteria of Alagille syndrome [20].

It is known that the Notch signaling pathway is important for regulation of angiogenesis [21]. Notch-1 has also been recognized as a $\mathrm{T}$ cell oncogene and is highly expressed in the thymus. Specifically, immature lymphoid cells that enter the thymus differentiate along the $\mathrm{B}$ cell lineage without Notch signals $[19,22]$. Notch-1 may 
have implications for further $\mathrm{T}$ cell lineage differentiation. In one mouse model, thymocytes with a functional $\gamma \delta \mathrm{T}$ cell receptor (TCR) were diverted to the $\alpha \beta$ lineage with a constitutive Notch-1 signal [22]. In addition to TCR lineage, Notch proteins also seem to regulate CD4 $\mathrm{T}_{\mathrm{H}}$ cell differentiation; however, there are reports of Notch signaling promoting both $\mathrm{T}_{\mathrm{H}^{-}}$and $\mathrm{T}_{\mathrm{H}^{-}}$- cell polarization [19]. CD4 $\mathrm{T}$ cells incubated with Jagged1-expressing antigen presenting cells (APC) were induced to a $\mathrm{T}_{\mathrm{H}^{-}}-2$ cell fate, and those incubated with APC that over-expressed Jagged1 were induced to a regulatory T cell fate [19]. Thus, JAG1 mutations, like those seen in Alagille syndrome, are likely to alter $\mathrm{T}$ cell development within the thymus and modify effector CD4 T cell differentiation in the peripheral lymphoid organs. Taken together with the evidence that over-expression of Jagged1 on APC promotes $\mathrm{T}_{\text {reg }}$ and $\mathrm{T}_{\mathrm{H}}-2$ CD4 $\mathrm{T}$ cell development [19] and intact Notch signaling is required for appropriate populations of TCR $\gamma \delta$ $\mathrm{T}$ cells and TCR $\alpha \beta \mathrm{T}$ cells $[19,22]$, then it follows that Jagged1/Notch interactions are critical to immune homeostasis.

The genetics of sarcoidosis are being investigated. There are candidate genes located on chromosomes 5 and 6. One such candidate gene is BTNL2 in the major histocompatibility complex II region on chromosome 6 [2]. Blau syndrome has been associated with mutations in CARD15 /NOD2 which results in a gain of function mutation; similar mutations have been demonstrated in cases of early onset sarcoidosis $[5,6]$. The CARD15/NOD2 mutations are associated with increased basal NF- $\mathrm{B}$ (nuclear factor $\kappa \mathrm{B}$ ) activity independent of muramyl dipeptide, a component of bacterial peptidoglycan, and a ligand for CARD15 in phagocytic cells [5]. The NF- $\mathrm{kB}$ signaling pathway is preferentially required for the production of interferon $\gamma$ (IFN $\gamma$ ) through a CD4 $\mathrm{T}_{\mathrm{H}^{-}}$cell mediated pathway [23].

Granulomas are formed by activation of $\mathrm{T}_{\mathrm{H}}-1$ cells and macrophages; thus, sarcoidosis is often considered a $\mathrm{T}_{\mathrm{H}^{-1}}$ mediated disease. Granulomas in early sarcoidosis express a strong $\mathrm{T}_{\mathrm{H}^{-1}}$ phenotype with production of interleukin-2 and IFN $\gamma$, which results in a positive feedback loop to recruit additional $\mathrm{T}_{\mathrm{H}^{-1}}$ cells and IFN $\gamma$ production [18,24]. Therefore, if CARD15/NOD2 mutations result in increased basal NF- $\mathrm{kB}$ activity, which predispose to a $\mathrm{T}_{\mathrm{H}^{-}}$- cell preference and production of IFN $\gamma$, the resultant phenotypes would have a diffuse granulomatous process $[5,18,23,24]$.

The child described herein has a mutation in JAG1 which results in a truncated protein and is predicted to result in abnormal or no Jagged1/Notch binding. Without this signaling, a predominance of $\mathrm{T}_{\mathrm{H}^{-1}}$ phenotype and uncontrolled TCR $\gamma \delta \mathrm{T}$ cells would be expected, which would be similar to the immune phenotype seen in Blau syndrome with a resultant diffuse granulomatous process [5].

\section{Conclusion}

In conclusion, this child has a previously unreported combination of Alagille syndrome and inflammatory multisystem granulomata consistent with sarcoidosis. In patients with unusual symptoms, consideration of rare conditions is warranted even when they have other known and seemingly unrelated rare conditions. Although this is the first published report of sarcoidosis and Alagille syndrome in the same child, a JAG1 mutation would seem to predispose a patient to immunologic dysregulation and the development of granulomas.

\section{Consent}

Written informed consent was obtained from the child's mother for publication of this case report and any accompanying images. A copy of the written consent is available for review by the Editor-in-Chief of this journal.

\section{Abbreviations}

ACE: Angiotensin converting enzyme; CARD15: Caspase recruitment domain 15; NOD2: Nucleotide binding oligomerization domain 2; ESR: Erythrocyte sedimentation rate; TCR: T cell receptor; APC: Antigen presenting cell; NF-kB: Nuclear factor $\mathrm{kB}$; IFNץ: Interferon $\gamma$.

\section{Competing interests}

The authors declare that they have no competing interests.

\section{Authors' contributions}

$M M$ and $M Z$ drafted the manuscript. TB and RQC were involved in the patient care, participated in the manuscript's design and coordination, and critically revised the manuscript. DK provided figures and assistance with histological data and assisted with manuscript revision. All authors have reviewed the final manuscript and gave approval for publication.

\section{Acknowledgement}

$\mathrm{RQC}$ is supported by the Arthritis Foundation, Alabama Chapter Endowed Chair in Pediatric Rheumatology. Divisional research is also supported by Dr. Joe B. and Cornelia B. LaRussa.

\section{Author details}

${ }^{1}$ Department of Pediatrics, Division of Rheumatology, University of Alabama at Birmingham, Birmingham, AL, USA. ${ }^{2}$ Department of Pediatrics, Division of Neonatology, University of Alabama at Birmingham, Birmingham, AL, USA. ${ }^{3}$ Department of Pathology, University of Alabama at Birmingham, Birmingham, AL, USA.

Received: 22 June 2012 Accepted: 20 August 2012

Published: 31 August 2012

\section{References}

1. Cimaz R, Ansell BM: Sarcoidosis in the pediatric age. Clin Exp Rheumatol 2002, 20:231-237.

2. Shetty AK, Gedalia A: Childhood sarcoidosis: A rare but fascinating disorder. Pediatr Rheumatol Online J 2008, 6:16.

3. Tomita H, Sato S, Matsuda R, Sugiura Y, Kawaguchi H, Niimi T, et al: Serum lysozyme levels and clinical features of sarcoidosis. Lung 1999, 177:161-167.

4. Cron RQ, Wallace CA, Sherry DD: Childhood sarcoidosis-does age of onset predict clinical manifestations? J Rheumatol 1997, 24:1654-1656.

5. Kanazawa N, Okafuji I, Kambe N, Nishikomori R, Nakata-Hizume M, Nagai S, et al: Early-onset sarcoidosis and CARD15 mutations with constitutive nuclear factor-kappaB activation: common genetic etiology with Blau syndrome. Blood 2005, 105:1195-1197. 
6. Rosé CD, Doyle TM, Mcllvain-Simpson G, Coffman JE, Rosenbaum JT, Davey $M P$, et al: Blau syndrome mutation of CARD15/NOD2 in sporadic early onset granulomatous arthritis. J Rheumatol 2005, 32:373-375.

7. Krantz ID, Piccoli DA, Spinner NB: Clinical and molecular genetics of Alagille syndrome. Curr Opin Pediatr 1999, 11:558-564.

8. Mueller RF: The Alagille syndrome (arteriohepatic dysplasia). J Med Genet 1987, 24:621-626.

9. Emerick KM, Rand EB, Goldmuntz E, Krantz ID, Spinner NB, Piccoli DA: Features of Alagille syndrome in 92 patients: frequency and relation to prognosis. Hepatology 1999, 29:822-829.

10. Wardrop D: Ockham's razor: sharpen or re-sheathe? J R Soc Med 2008, 101:50-51.

11. Takemura T, Shishiba T, Akiyama O, Oritsu M, Matsui Y, Eishi Y: Vascular involvement in cutaneous sarcoidosis. Pathol Int 1997, 47:84-89.

12. Wei CH, Huang YH, Shih YC, Tseng FW, Yang CH: Sarcoidosis with cutaneous granulomatous vasculitis. Australas I Dermatol 2010, 51:198-201.

13. Petri $M$, Barr E, Cho K, Farmer E: Overlap of granulomatous vasculitis and sarcoidosis: presentation with uveitis, eosinophilia, leg ulcers, sinusitis, and past foot drop. J Rheumatol 1988, 15:1171-1173.

14. García-Porrúa C, González-Gay MA, García-País MJ, Blanco R: Cutaneous vasculitis: an unusual presentation of sarcoidosis in adulthood. Scand J Rheumatol 1998, 27:80-82.

15. Kavukçu S, Demir K, Soylu A, Anal O, Saatçi O, Göktay Y: A case of Takayasu disease with findings of incomplete Alagille syndrome. Rheumatol Int 2005, 25:555-557.

16. Online Mendelian Inheritance in Man, $O M M^{\circledR}$. Baltimore, MD: Johns Hopkins University; MIM Number: \{118450- ALAGILLE SYNDROME 1; ALGS1\}: \{02/21/ 2012\}. World Wide Web URL: http://omim.org/.

17. Sharma OP: Vitamin D, calcium, and sarcoidosis. Chest 1996, 109:535-539.

18. Lagana SM, Moreira RK, Lefkowitch JH: Hepatic granulomas: pathogenesis and differential diagnosis. Clin Liver Dis 2010, 14:605-617.

19. Osborne BA, Minter LM: Notch signalling during peripheral T-cell activation and differentiation. Nat Rev Immunol 2007, 7:64-75.

20. Eldadah ZA, Hamosh A, Biery NJ, Montgomery RA, Duke M, Elkins R, et al Familial Tetralogy of Fallot caused by mutation in the jagged 1 gene. Hum Mol Genet 2001, 10:163-169.

21. Gridley T: Notch signaling in vascular development and physiology. Development 2007, 134:2709-2718.

22. Deftos ML, Bevan MJ: Notch signaling in T cell development. Curr Opin Immunol 2000, 12:166-172.

23. Aronica MA, Mora AL, Mitchell DB, Finn PW, Johnson JE, Sheller JR, et al Preferential role for NF-kappa B/Rel signaling in the type 1 but not type 2T cell-dependent immune response in vivo. J Immunol 1999, 163:5116-5124.

24. Gerke AK, Hunninghake G: The immunology of sarcoidosis. Clin Chest Med 2008, 29:379-390. vii.

doi:10.1186/1546-0096-10-32

Cite this article as: Mannion et al: Sarcoidosis in a young child with Alagille syndrome: a case report. Pediatric Rheumatology 2012 10:32.

\section{Submit your next manuscript to BioMed Central and take full advantage of:}

- Convenient online submission

- Thorough peer review

- No space constraints or color figure charges

- Immediate publication on acceptance

- Inclusion in PubMed, CAS, Scopus and Google Scholar

- Research which is freely available for redistribution

Submit your manuscript at www.biomedcentral.com/submit
Biomed Central 